\title{
Spin Exchange and Surface Relaxation in the Atomic Hydrogen Maser
}

\section{Citation}

Berg, Howard C. 1965. Spin exchange and surface relaxation in the atomic hydrogen maser.

Physical Review 137(6A): A1621-A1634.

\section{Published Version}

http://dx.doi.org/10.1103/PhysRev.137.A1621

\section{Permanent link}

http://nrs.harvard.edu/urn-3:HUL.InstRepos:3219931

\section{Terms of Use}

This article was downloaded from Harvard University's DASH repository, and is made available under the terms and conditions applicable to Other Posted Material, as set forth at http:// nrs.harvard.edu/urn-3:HUL.InstRepos:dash.current.terms-of-use\#LAA

\section{Share Your Story}

The Harvard community has made this article openly available.

Please share how this access benefits you. Submit a story.

Accessibility 


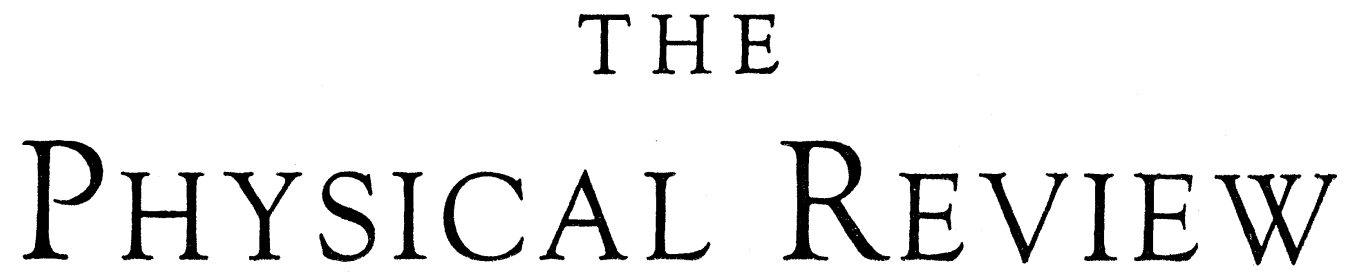

A journal of experimental and theoretical physics established by E. L. Nichols in 1893

Second Series, Vol. 137, No. 6A

15 MARCH 1965

\title{
Spin Exchange and Surface Relaxation in the Atomic Hydrogen Maser*
}

\author{
HOWARD C. BERG $\dagger$ \\ Lyman Laboratory of Physics, Harvard University, Cambridge, Massachusetts \\ (Received 12 August 1964; revised manuscript received 6 November 1964)
}

\begin{abstract}
An experiment using the atomic-hydrogen maser is described which confirms several predictions of the theory of spin exchange and which provides new information on the spin relaxation of hydrogen at solid surfaces. Atoms in the $\left(F=1, m_{F}=0\right)$ state, which are confined to a storage bulb, are put into a coherent radiating state by a microwave pulse at the $\Delta m_{F}=0$ hyperfine frequency. The initial amplitude and the decay rate of the induced signal are measured. Atoms or molecules containing unpaired electrons are added to the storage bulb at various constant rates, and the pulse measurements are repeated. In this manner, the rate at which spin-exchange collisions decrease the oscillating dipole movement of the radiating gas, a $T_{2}$ process, is compared with the rate at which the same collisions decrease the population difference $(F=1$, $\left.m_{F}=0\right)-\left(F=0, m_{F}=0\right)$, a $T_{1}$ process. It is shown for a spin-exchange theory which neglects spin-orbit coupling and all direct magnetic interactions that the ratio of the relaxation times, $T_{2} / T_{1}$, is independent of all collision parameters. For collisions between atomic hydrogen and atomic deuterium, nitric oxide, and molecular oxygen, the predicted $T_{2} / T_{1}$ ratios are $4 / 3$. For hydrogen-hydrogen collisions, the predicted $T_{2} / T_{1}$ ratio is 2 . The deuterium, nitric oxide, and oxygen results agree with the theory, but the hydrogen results are at least $5 \%$ low. The magnitude of the discrepancy depends upon the composition of the storage-bulb wall, and the error is explained by a wall relaxation process which has a rate proportional to the hydrogen density. A second wall relaxation process is observed with a rate independent of the hydrogen density, but which increases rapidly with temperature. Both wall relaxation processes appear to be related to the chemical reactivity of atomic hydrogen. These phenomena are examined in detail for a series of hydrocarbon, fluorocarbon, and mixed hydro-fluorocarbon surfaces. Approximate experimental hydrogen-radiation decay-rate cross sections also are given for a series of molecular gases.
\end{abstract}

\section{INTRODUCTION}

$\mathrm{T}^{\mathrm{H}}$ HIS paper describes a series of spin-relaxation experiments performed with the atomic hydrogen maser $^{1-5}$ in which atoms of hydrogen in the ground electronic state are allowed to collide with other atoms or molecules containing unpaired electrons. The dominant mechanism leading to a change of spin state in this case is spin exchange, ${ }^{6-8}$ a process having as its physical

* Work supported by the National Science Foundation and the Office of Naval Research.

$\dagger$ Junior Fellow, Society of Fellows; National Institutes of Health Predoctoral Fellow, 1959-1963.

${ }^{1} \mathrm{H}$. M. Goldenberg, D. Kleppner, and N. F. Ramsey, Phys. Rev. Letters 5, 361 (1960).

${ }^{2}$ H. M. Goldenberg, thesis, Harvard University, 1960 (unpublished).

${ }^{3}$ D. Kleppner, H. M. Goldenberg, and N. F. Ramsey, Appl. Opt. 1, 55 (1962).

${ }^{4}$ D. Kleppner, H. M. Goldenberg, and N. F. Ramsey, Phys. Rev. 126, 603 (1962).

${ }^{5}$ N. F. Ramsey, IRE Trans. Instr. I-11, 177 (1962).

6 E. M. Purcell and G. B. Field, Astrophys. J. 124, 542 (1956).

7 J. P. Wittke, thesis, Princeton University, 1955 (unpublished).

8 J. P. Wittke and R. H. Dicke, Phys. Rev. 103, 620 (1956). basis the identity of the electrons. The change in spin state of hydrogen can be inferred from changes in the radiation field of the maser. If the maser is used in a pulsed mode, it is possible to compare with some precision the rate at which spin-exchange collisions reduce the oscillating magnetic dipole moment of the hydrogen gas with the rate at which they decrease the population difference of the stationary states between which the radiative transition occurs. There is a single relaxation time associated with each of these processes. The ratio of these times, $T_{2} / T_{1}$, is a number which is independent of the absolute collision rate and of the detailed shape of the interaction potentials. If the $T_{2} / T_{1}$ ratio is measured, the validity of the spin-exchange theory can be tested without reference to the absolute values of any collision parameters.

The first purpose of the present work was to test the predictions of the spin-exchange theory by measuring the $T_{2} / T_{1}$ ratios for collisions between atomic hydrogen and atomic hydrogen, atomic deuterium, nitric oxide (a ${ }^{2} \mathrm{II}$ diatomic molecule), and molecular oxygen (a ${ }^{3} \Sigma$ 
diatomic molecule). The results are of particular interest in view of the apparent discrepancy between theory and experiment in the measurement of spinexchange (frequency shift)/(line broadening) ratios for collisions between free electrons and alkali metals. ${ }^{9-11}$ The (frequency shift)/(line broadening) ratios are very sensitive to the shape of the interaction potentials, and interpretation of the results requires detailed computation of partial-wave phase shifts, a complication not met here.

The $T_{2} / T_{1}$ experiments demonstrate clearly that spin exchange is a coherent process. Part of the phase information associated with the knowledge of the instantaneous oscillating magnetic moment of the radiating gas is preserved even if each atom undergoes an exchange collision of arbitrary strength. The degree of coherence is reflected in the value $T_{2}$. For the $\left(F=1, m_{F}=0\right) \rightarrow\left(F=0, m_{F}=0\right)$ transition of hydrogen, the theoretical $T_{2} / T_{1}$ ratio for hydrogen-hydrogen collisions is 2 and the theoretical $T_{2} / T_{1}$ ratio for hydrogen-nonhydrogen collisions is $4 / 3$. The difference arises from the fact that phase information can be passed from atom to atom. A hydrogen atom initially in a stationary state can be left in a radiating state following an exchange collision with another hydrogen atom initially in a radiating state.

The second purpose of the present work was to learn more of the nature and extent of spin relaxation at the maser storage bulb wall. The hydrogen maser utilizes a storage technique in which the atoms are confined to a bulb in the center of a microwave cavity. During the time that the atoms interact with the cavity field, they undergo many thousands of collisions with the bulb wall. The relaxation at the wall must be known to interpret the spin-exchange results, to understand in detail the function and limitations of the maser, and to learn how the storage technique might be improved. The experiments have been performed with walls coated with Teflon and with the surface prepared by the reaction of dimethyldichlorosilane with fused quartz. This aspect of the work is related to relaxation rate measurements made in buffer-gas-free optical pumping experiments, especially by Bouchiat, ${ }^{12}$ who has studied the relaxation of $\mathrm{Rb}$ on hydrocarbon and deuterated hydrocarbon surfaces, and to the broken-atomic-beam experiment of Goldenberg, Kleppner, and Ramsey. ${ }^{13}$

Spin-relaxation measurements with the maser have a beauty inherent in their simplicity: The structure of atomic hydrogen is well known; hyperfine transitions are detected directly by monitoring the radiation field; relaxation due to magnetic field inhomogeneities can be

L. C. Balling, R. J. Hanson, and F. M. Pipkin, Phys. Rev. 133 A607 (1964).

${ }^{10}$ P. L. Bender, Phys. Rev. 134, A1174 (1964).

${ }^{11}$ L. C. Balling and F. M. Pipkin, Phys. Rev. 136, A46 (1964).

12 M. A. Bouchiat, J. Phys. Radium 24, 379, 611 (1963); thesis, Université de Paris, 1964 (unpublished).

${ }^{13}$ H. M. Goldenberg, D. Kleppner, and N. F. Ramsey, Phys. Rev. 123, 530 (1961). eliminated; the density of hydrogen and the gas under study can be varied quickly and independently; and the storage times are sufficiently long that signal intensities and decay rates can be measured readily to an accuracy of about $1 \%$.

This paper is organized in the following way. In Sec. II the nature of the experiment is outlined and the assumptions on which the spin-exchange theory is based are reviewed. In Sec. III the experimental techniques are described. In Sec. IV the results of the experiment are discussed. In the final section the implications of the work are summarized. ${ }^{14} \mathrm{~A}$ detailed description of the design and construction of hydrogen masers ${ }^{15}$ and a detailed discussion of the analysis of exponentially decaying pulsed signals ${ }^{16}$ will be given elsewhere.

\section{THEORY OF THE EXPERIMENT}

We are concerned with transitions which occur between the four hyperfine states of $1 S$ atomic hydrogen, which at low magnetic fields have the quantum numbers $\left(F, m_{F}\right)=(1,1),(1,0),(1,-1)$, and $(0,0)$, which we label $1,2,3$, and 4 , respectively. The experimental arrangement is illustrated in Fig. 1. (The vacuum envelope and the inlets for admitting gases other than atomic hydrogen and deuterium are not shown.) The left part of the drawing represents the hydrogen maser as previously described. ${ }^{1-5}$ Atomic hydrogen is generated in a radiofrequency discharge and passes through an inhomogeneous state-selecting magnetic field. This field focuses atoms in the states 1 and 2 into a coated fused quartz bulb centered in a cylindrical cavity. The cavity operates in a $T E_{011}$ mode and is tuned to the $\Delta m_{F}=0$ hyperfine frequency at approximately $1420.405 \mathrm{Mc} / \mathrm{sec}$. There is a fixed magnetic field along the axis of the cavity of several milligauss, maintained by a solenoid and a series of magnetic shields (not shown). The atoms are confined to the bulb for the order of $1 \mathrm{sec}$, during which time they make about $10^{5}$ collisions with the wall, retaining to a large extent their initial hyperfine states. In the present experiment the hydrogen-beam flux is small enough that self-excited maser oscillation does not occur, and the probability that two hydrogen atoms collide with one another before leaving the bulb is

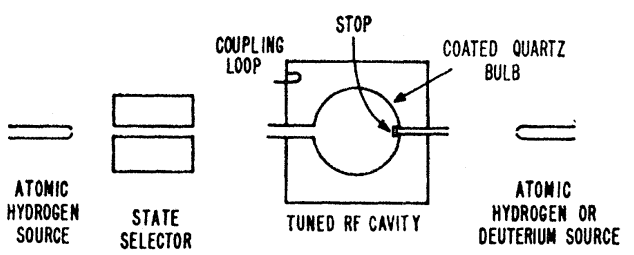

Fig. 1. Schematic of the atomic hydrogen maser modified for spin-relaxation studies.

${ }^{14}$ H. C. Berg, thesis, Harvard University, 1964 (unpublished).

${ }^{15}$ D. Kleppner, H. C. Berg, S. B. Crampton, N. F. Ramsey, R. F. C. Vessot, H. E. Peters, and J. Vanier (to be published). ${ }^{16} \mathrm{H}$. C. Berg (to be published). 
negligible. The pressure in the vacuum chamber connected to the bulb is about $5 \times 10^{-8} \mathrm{~mm} \mathrm{Hg}$ with the beam on, and the background gas is largely $\mathrm{H}_{2}$ and $\mathrm{N}_{2}$, which have extremely small hydrogen spin-relaxation cross sections. ${ }^{17}$

If a short pulse at the $\Delta m_{F}=0$ transition frequency is delivered to the cavity, a signal is observed which decays exponentially in time. (This is true provided the flux of polarized hydrogen is sufficiently small; the decay rate is then independent of the power level. The amplitude of the signal is directly proportional to the oscillating dipole moment of the hydrogen gas. $\left.{ }^{4,14}\right)$ The decay constant of this signal is $\left(T_{e 1}{ }^{-1}+T_{w 2}{ }^{-1}\right) \mathrm{sec}^{-1}$, the sum of the rate $T_{e 1}^{-1}=T_{e 2}^{-1}$ at which atoms escape from the bulb and the rate $T_{w 2}{ }^{-1}$ at which they are relaxed by the wall. ${ }^{18}$ The initial height of the signal is a measure of the population density difference of atoms in the states 2 and 4 present in the bulb at the time of the pulse $n_{0}$ and the observed signal is proportional to $n_{0} \exp \left[-t\left(T_{e 1}{ }^{-1}+T_{w 2}{ }^{-1}\right)\right]$, where $t$ is the time [Fig. 2(a)].

Spin-exchange relaxation is induced by adding to the bulb at a constant rate atoms or molecules taken from a source at thermal equilibrium. The right side of Fig. 1 illustrates the arrangement for the study of hydrogen and deuterium. A second entrance has been added to the storage bulb in line with a second radiofrequency discharge. This is a narrow coated tube chosen so that no more than $2 \%$ of the atoms may leave the bulb by this route. Nitric oxide and oxygen are added directly to the maser vacuum chamber and enter the bottle through the first entrance. In the measurement of absolute relaxation cross sections of gases, the second discharge is replaced by a gas-handling system. During the course of the addition of any of these gases, the maser itself is not disturbed; the influx of polarized hydrogen remains unchanged.

Spin-exchange collisions with the added gas reduce the steady-state population difference $n_{0}$ to a smaller value $n$ and increase the radiation decay rate to a larger value, $\left(T_{e 1}^{-1}+T_{w 2}{ }^{-1}+T_{\theta 2}{ }^{-1}\right) \mathrm{sec}^{-1}$. When the system is pulsed, the signal has a smaller initial height and decays more rapidly in time [Fig. 2(b)]. The fractional change in the initial height measures the effect of spin-exchange collisions in establishing a new stationary-state population difference, and this process occurs in the absence of a radiation field. The change in the decay rate measures the effect of spin-exchange collisions in reducing the oscillating dipole moment of the hydrogen gas. The value of $T_{g 2} / T_{g 1}$ can be found from the study of the relation between these two changes for a series of added-gas pressures.

${ }^{17}$ L. W. Anderson, F. M. Pipkin, and J. C. Baird, Phys. Rev, Letters 1, 229 (1958); 4, 69 (1960); Phys. Rev. 120, 1279 (1960).

18 We use the subscript 1 when referring to a depolarization time and the subscript 2 when referring to a radiation-field decay time. The letter subscripts identify the relaxation process: $e=$ excape, $w=$ wall collisions, $g=$ added gas collisions.

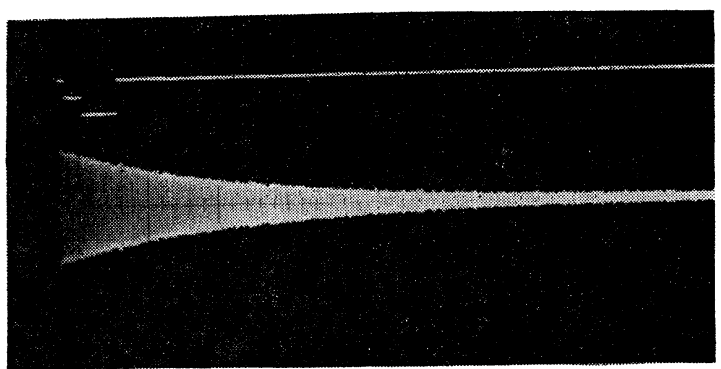

(a)

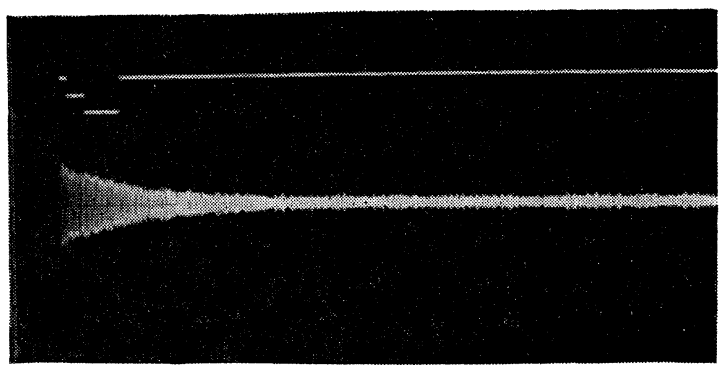

(b)

FIG. 2. (a) Decay of the maser signal without added gas. (The segments of the upper trace are $0.02,0.05$, and $0.10 \mathrm{sec}$ long, respectively. The carrier is $5.76 \mathrm{kc} / \mathrm{sec}$.) (b) As (a) except for the addition of unpolarized hydrogen and an increase in i.f. amplifier gain by a factor 1.8 .

In practice, a control experiment is required in which relaxation is induced by a known process. This is accomplished by adding a third exit to the bulb, a trap door which can be manipulated from outside the vacuum chamber, allowing atoms to escape at varying rates. In principle this relaxation process is the least subtle: Atoms are physically removed from the ensemble $\left(T_{e 2} / T_{e 1}=1\right)$.

The general form of the $T_{2} / T_{1}$ relationship will now be derived. The expression for the total time rate of change of the hydrogen density matrix includes a term dictated by the free-atom Hamiltonian, a flow term which describes the influx of the atom beam(s) and the rate at which the atoms escape from the bulb, a wallrelaxation term, and a term due to spin-exchange collisions. The oscillating magnetic field following the pulse is so small that for all subsequent times of interest, the term which describes the time rate of change of the density matrix due to the interaction of the atoms and the field can be neglected. Under the conditions of the experiment, changes due to the motion of atoms in an inhomogeneous magnetic field are also negligible. The initial amplitude of the pulsed signal is proportional to the steady-state value of $n_{H}\left(H_{22}-H_{44}\right)$, where $n_{H}$ is the total hydrogen density, and $H_{i j}$ is the $i j$ element of the hydrogen density matrix.

With the added-gas source off and at hydrogen densities at which hydrogen-hydrogen spin-exchange 
relaxation is negligible compared to the escape rate $T_{e 1}{ }^{-1}$,

$$
\begin{aligned}
\frac{d}{d t}\left(H_{22}-H_{44}\right)=\frac{1}{I T_{e 1}}\left(I_{2}-I_{4}\right)- & \frac{1}{T_{e 1}}\left(H_{22}-H_{44}\right) \\
& -\frac{1}{T_{w 1}}\left(H_{22}-H_{44}\right),
\end{aligned}
$$

where $I_{j}$ is the number of hydrogen atoms entering the bulb per unit time in the state $j$ and $I=I_{1}+I_{2}+I_{3}+I_{4}$. In treating the wall relaxation phenomenologically, we assume that it can be characterized by a single $T_{1}$ and a single $T_{2}$ relaxation time. ${ }^{19}$

The steady-state value of $n_{H}\left(H_{22}-H_{44}\right)$, which we define to be $n_{0}$, follows at once from the condition $(d / d t)\left(H_{22}-H_{44}\right)=0$.

$$
n_{0}=\left(I_{2}-I_{4}\right) / V_{b}\left(T_{e 1}^{-1}+T_{w 1}{ }^{-1}\right),
$$

where we have used the relation $n_{H}=I T_{e 1} / V_{b}$, where $V_{b}$ is the volume of the storage bulb.

After the pulse, the radiation decay rate can be computed from the time rate of change of the density matrix element $H_{24}$ or $H_{42}$. With the added gas source off,

$$
\frac{d}{d t} H_{24}=-\frac{1}{T_{e 1}} H_{24}-\frac{1}{T_{w 2}} H_{24}-i \omega_{24} H_{24}
$$

where $\omega_{24}$ is $2 \pi$ times the hyperfine frequency of the free atom, and we ignore any terms associated with a wall frequency shift. Equation (3) can be solved by the substitution $H_{24}(t)=H_{24}(0) \exp (-i \omega t)$. The amplitude of the signal, which is proportional to $H_{24}(0)$, decays exponentially at the rate $T_{e 1}{ }^{-1}+T_{w 2}{ }^{-1}$.

Let atomic deuterium be added to the storage bulb at a rate such that the total deuterium density is $n_{D}$. The time rate of change of the hydrogen density matrix elements due to hydrogen-deuterium spin-exchange collisions are given in the matrix of Table VII of the paper of Balling, Hanson, and Pipkin. ${ }^{9}$ (The use in the deuterium example of the results derived there for free electrons is justified below.) We must add to the right side of Eq. (1) the spin-exchange term $-n_{\mathrm{D}}\left\langle v_{\mathrm{DH}} \sigma_{\mathrm{SF}}\right\rangle_{\mathrm{av}}$ $\times\left(H_{22}-H_{44}\right)$, where $v_{D H}$ is the relative deuteriumhydrogen velocity, $\sigma_{\mathrm{SF}}$ is the spin-flip cross section defined in Ref. 9, Eq. (25), and the average is over the relative velocity $v_{\mathrm{DH}}$. For the new steady-state popu-

${ }^{19}$ It is sufficient that the wall-relaxation process induces transitions between the hydrogen states $i$ and $j$ at a rate $T_{i j}{ }^{-1}$ such that $T_{i j}=T_{k l}$ for $i j, k l=21,41 ; 23,43 ; 24,42 ; 12,14$; and 32,34 . In this case $T_{1}^{-1}=T_{21}{ }^{-1}+T_{23}{ }^{-1}+2 T_{24}{ }^{-1}$, and the four-level problem reduces to a two-level problem characterized by a single relaxation time. This is true for hydrogen-nonhydrogen spin-exchange collisions, for hydrogen-hydrogen spin-exchange collisions, if the atoms are treated as distinguishable, for any process for which the $T_{i j}$ are equal (complete randomization of spin state), and for any process which removes atoms from the bulb (chemical reaction). lation-density difference $n$, we find

where

$$
n=\frac{\left(I_{2}-I_{4}\right)}{V_{b}\left(T_{e 1}{ }^{-1}+T_{w 1} 1^{-1}+T_{g 1^{-1}}\right)},
$$

$$
T_{g 1}{ }^{-1}=n_{D}\left\langle v_{\mathrm{DH}} \sigma_{\mathrm{SF}}\right\rangle_{\mathrm{av}} \text {. }
$$

To the right side of Eq. (3) we must add the term $-\left(\frac{3}{4} n_{\mathrm{D}}\right)\left\langle v_{\mathrm{DH}} \sigma_{\mathrm{SF}}\right\rangle_{\mathrm{av}} H_{24}$, and the pulsed signal decays exponentially at a rate $T_{e 1}{ }^{-1}+T_{w 2}{ }^{-1}+T_{g 2}{ }^{-1}$, where ${ }^{20}$

$$
T_{g 2}{ }^{-1}=\frac{3}{4} n_{\mathrm{D}}\left\langle v_{\mathrm{DH}} \sigma_{\mathrm{SF}}\right\rangle_{\mathrm{av}} .
$$

Dividing Eq. (2) by Eq. (4), we find

$$
\frac{n_{0}}{n}=\frac{T_{g 1}^{-1}}{T_{e 1}^{-1}+T_{w 1}^{-1}}+1
$$

which can be written in the form

$$
\frac{n_{0}}{n}=\left[1+\left(\frac{T_{w 2}}{T_{w 1}}-1\right) \frac{T_{0}}{T_{w 2}}\right]^{-1}\left(\frac{T_{g 2}}{T_{g 1}}\right) \frac{T_{0}}{T_{g 2}}+1
$$

where $T_{0}^{-1}=T_{w 2}{ }^{-1}+T_{e 1}{ }^{-1}$ is the decay rate observed with the added-gas source off. The value in square brackets depends on the nature of the wall relaxation, i.e., on the value $T_{w 2} / T_{w 1}$, and on the magnitude of the wall relaxation relative to the escape rate $T_{e 1}{ }^{-1}$. It is independent of the added-gas density provided the added gas does not change the structure of the wall. Pulsing with the added gas off provides a measurement of $n_{0}$ and $T_{0}^{-1}$. Pulsing with the added gas on provides a measurement of $n$ and $T_{0}^{-1}+T_{g 2}{ }^{-1}$. In the present example $T_{g 2}{ }^{-1}$ is proportional to $n_{D}$. If the pulse measurements are made for a series of deuterium pressures and the values of $n_{0} / n$ are plotted as a function of $T_{0} / T_{g 2}$, then we should obtain a straight line which passes through the point $n_{0} / n=1$ and $T_{0} / T_{g 2}=0$ and which has the slope

$$
\left[1+\left(\frac{T_{w 2}}{T_{w 1}}-1\right) \frac{T_{0}}{T_{w 2}}\right]^{-1}\left(\frac{T_{g 2}}{T_{g 1}}\right) .
$$

Opening a trap door and increasing $T_{e 1}{ }^{-1}$ is equivalent to adding a gas for which $T_{2} / T_{1}=1$. The slope for the trap-door experiment is just the factor $\left[1+\left(T_{w 2} / T_{w 1}-1\right) T_{0} / T_{w 2}\right]^{-1}$, a number which is less, equal, or greater than 1 if $T_{w 2} / T_{w 1}$ is greater, equal, or less than 1 , respectively. Once this factor is known, $T_{g 2} / T_{g 1}$ can be found for any added gas which does not change the structure of the wall.

The escape rate $T_{e 1}{ }^{-1}$ is a function of the geometry of the storage bulb, the average velocity of the hydrogen atoms, and the nature of the reflection of atoms from the walls. If this reflection is diffuse, and the dwell time

${ }^{20}$ The 1,$0 ; 0,0$ and 0,$0 ; 1,0$ elements of the matrix of Table VII, Ref. 9, should read $-3 \mathrm{H}_{24}$ and $-3 \mathrm{H}_{42}$, respectively; the other terms average to zero. 
TABLE I. The matrix $\boldsymbol{\sigma}(H) \cdot \boldsymbol{\sigma}\left(0_{2}\right)$. The state labels are written so that the first number gives the state of the hydrogen atom, and the second number gives the $m_{S}$ value for the spin-1 system. The elements not shown are zero.

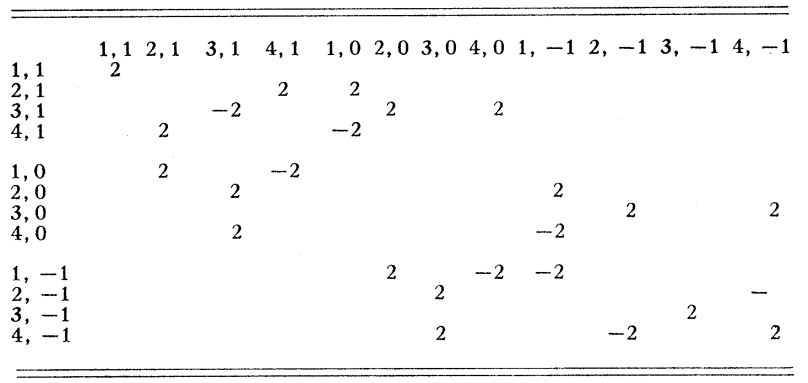

of the atoms on the wall is negligible compared to the time required for them to cross the bulb, then $T_{e 1}{ }^{-1}$ can be calculated from the equations of molecular flow. ${ }^{21,22}$ Since $T_{w 2}{ }^{-1}=T_{0}{ }^{-1}-T_{e 1}{ }^{-1}$, the slope of the trap-door experiment provides an estimate of $T_{w 2} / T_{w 1}$. We can learn something of the extent and nature of relaxation at the wall.

If atoms are completely removed by chemical reaction, the $T_{2} / T_{1}$ value is 1 . If the relaxation process removes atoms from the ensemble and returns them with equal probability to each of the hyperfine states, the $T_{2} / T_{1}$ value is 1 . If only the phase of the radiating atom is randomized, the $T_{2} / T_{1}$ value is $0 .{ }^{23}$

From Eqs. (5) and (6) we find for deuterium $T_{g 2} / T_{g 1}$ $=4 / 3$. This result is independent of the spin-flip cross section $\sigma_{\mathrm{SF}}$, of the distribution of velocities $v_{\mathrm{DH}}$, and of the deuterium electron spin polarization. The same result is expected for collisions with the molecule $\mathrm{NO}$, which contains a single unpaired electron.

The hydrogen-hydrogen result can be computed in similar fashion using the matrix of Table X, Ref. 9, with the result

$$
\begin{aligned}
& T_{g 1}{ }^{-1}=n_{\mathrm{H}}\left\langle v_{\mathrm{HH}}\left[\sigma_{\mathrm{SF}}+\left(2 H_{22}+2 H_{44}-1\right) \sigma_{\mathrm{SF}^{\prime}}\right]\right\rangle_{\mathrm{av}}, \\
& T_{g 2}{ }^{-1}=\frac{1}{2} n_{\mathrm{H}}\left\langle v_{\mathrm{HH}}\left[\sigma_{\mathrm{SF}}+\left(2 H_{22}+2 H_{44}-1\right) \sigma_{\mathrm{SF}^{\prime}}\right]\right\rangle_{\mathrm{av}},
\end{aligned}
$$

where $\sigma_{\mathrm{SF}}$ and $\sigma_{\mathrm{SF}^{\prime}}$ are defined in Eqs. (B3) and (B5), Ref. 9. In deriving these rates we have assumed that the added hydrogen is unpolarized, and we have neglected the term proportional to $i$ which, when added to the right side of Eq. (3), gives rise to a small frequency shift. Provided $\left(2 \mathrm{H}_{22}+2 \mathrm{H}_{44}-1\right)$ is not changed by pulsing, $T_{g 2} / T_{g 1}=2$. The quantity $\left(H_{22}+H_{44}\right)$ is the probability that an atom will be found either in the state 2 or the state 4 , a probability which is unchanged by inducing a transition between the states 2 and 4 . The spinexchange time-rate-of-change expression for $\left(H_{22}+H_{44}\right)$, however, contains a term of the form $\frac{1}{2} n_{\mathrm{H}} v_{\mathrm{HH}}\left(\sigma_{\mathrm{SF}}-\sigma_{\mathrm{SF}^{\prime}}\right)$

\footnotetext{
${ }^{21}$ P. Clausing, Ann. Physik 12, 961 (1932).

${ }_{22} \mathrm{~S}$. Dushman, Scientific Foundations of Vacuum Technique (John Wiley \& Sons, Inc., New York, 1962), 2nd ed., pp. 90-95.

${ }_{23}$ This value would be observed, for example, if transitions were induced with equal probability between the states 1,2 and 2,3 in the special case $I_{1}=I_{2}=I_{3}$ and $I_{4}=0$.
}

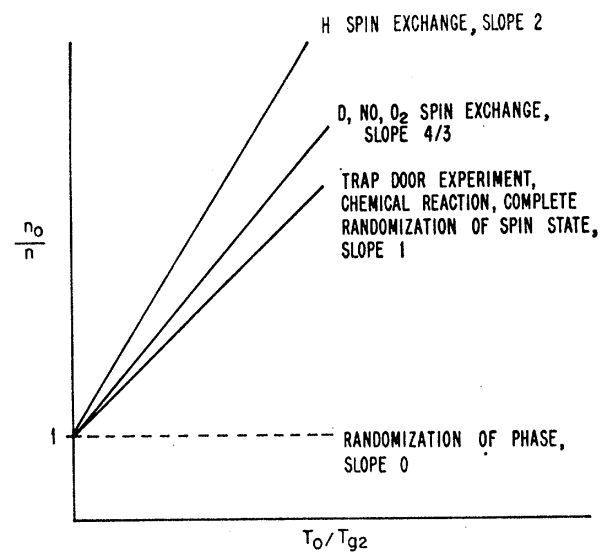

FIG. 3. Theoretical dependence of the initial signal height ratio $n_{0} / n$ on the relative radiation decay rate increment $T_{0} / T_{g_{2}}$ in the event $T_{w 2}{ }^{-1}=0$ or $T_{w 2} / T_{w 1}=1$.

$\mathrm{H}_{24} \mathrm{H}_{42}$, and the complete steady-state equation has not been solved. In practice, the unpolarized hydrogenbeam flux is about 150 times larger than the stateselected beam flux, $\left(H_{22}+H_{44}\right) \simeq \frac{1}{2}$, and the $\sigma_{\mathrm{SF}^{\prime}}$ term cannot be observed. (The $\sigma_{\mathrm{SF}^{\prime}}$ term does not appear in the form of the theory in which the atoms are considered distinguishable.)

The $\boldsymbol{\sigma}(\mathrm{H}) \cdot \boldsymbol{\sigma}\left(\mathrm{O}_{2}\right)$ matrix for collisions between hydrogen and a system with two unpaired electrons, for which the spin angular-momentum quantum number is 1 , is given in Table I. Using this matrix in the equations of Ref. 9, we find that the $T_{g 1}$ times depend upon the oxygen electron spin alignment, but that the $T_{g 2}$ time is independent of the alignment. If the molecules are in the spin states $m_{S}= \pm 1$, then

$$
T_{g 1}{ }^{-1}=\frac{2}{3} n_{\mathrm{O}_{2}}\left\langle\nu_{\mathrm{HO}_{2}} \sigma_{\mathrm{SF}}\right\rangle_{\mathrm{av}} \text {. }
$$

If they are in the spin state $m_{S}=0$, then

$$
T_{g 1}{ }^{-1}=(8 / 9) n_{\mathrm{O}_{2}}\left\langle v_{\mathrm{HO}_{2}} \sigma_{\mathrm{SF}}\right\rangle_{\mathrm{av}} \text {. }
$$

In practice, oxygen is not aligned. The electron spin is coupled to the rotational angular momentum of the molecule, thousands of oxygen-wall collisions occur for every oxygen-hydrogen exchange collision, and the spin state is continuously randomized. In this case,

$$
T_{g 1}^{-1}=(32 / 27) n_{\mathrm{O}_{2}}\left\langle v_{\mathrm{HO}_{2}} \sigma_{\mathrm{SF}}\right\rangle_{\mathrm{av}} \cdot
$$

In all cases,

$$
T_{g 2}{ }^{-1}=(8 / 9) n_{\mathrm{O}_{2}}\left\langle v_{\mathrm{HO}_{2}} \sigma_{\mathrm{SF}}\right\rangle_{\mathrm{av}} .
$$

From Eqs. (14) and (15) we obtain the predicted slope $4 / 3$ for the oxygen experiment.

The results predicted by the theory for the various relaxation experiments are summarized in Fig. 3, where the slopes given are those valid in the event $T_{w 2} / T_{w 1}=1$ or $T_{w 2}{ }^{-1}=0$.

We have quoted from the results of the partial-wave formulation of the spin-exchange theory begun by 
Dalgarno ${ }^{24}$ and extended by Balling, Hanson, and Pipkin..$^{9,25}$ The results can be derived with equal generality in the semiclassical formulation of Purcell and Field ${ }^{6}$ and Wittke and Dicke. ${ }^{7,8}$ The electron-spinstate transformation operator used in this formulation, which when applied to the initial electron spin state generates the final electron spin state, is, in fact, the most general unitary electron-spin transformation consistent with the equations of motion for a Hamiltonian which commutes with the total spin angular momentum $\mathbf{S}=\frac{1}{2} \hbar\left(\boldsymbol{\sigma}_{1}+\boldsymbol{\sigma}_{2}\right)$, where $\boldsymbol{\sigma}_{1}$ and $\boldsymbol{\sigma}_{2}$ are the Pauli electronspin operators for the two colliding systems. This transformation is of the form

$$
U=\sum_{S} e^{i \phi_{S} P_{(S)}}
$$

where $P_{(S)}$ is the projection operator for the state of total spin $S$, and $\phi_{S}$ is a number which depends on the dynamics of the collision. The theory is semiclassical because explicit evaluation of phase shifts of the form $\phi_{1}-\phi_{2}$ requires the assumption of a classical path, but the $T_{2} / T_{1}$ results depend only on the structure of the transformation and not on the specific values $\phi_{S}$.

In using a transformation of this kind, it is assumed that spin-orbit coupling, the magnetic coupling between the electrons and the nuclei, the coupling between the electrons and the external magnetic field, and the tensor part of the electron dipole-dipole coupling can be neglected during the collision. Under these assumptions, the outcome of the collision depends only on the electron spin of the added gas, and the results predicted for free electrons, deuterium atoms, and nitric oxide molecules are the same. The proton spin state is not changed by the collision provided the collision period is small compared to the period of precession of the proton in the magnetic field of the electron, which is the period of the hyperfine frequency, $7 \times 10^{-10}$ sec. (Since a hydrogen atom at room temperature travels about $3 \AA$ in $10^{-13} \mathrm{sec}$, this condition is well satisfied.) These are the assumptions tested by the present experiment.

The operator $U$ may be written in the form $a+b \sigma_{1} \cdot \sigma_{2}$. For the spin- $\frac{1}{2}$ case, $a=\frac{1}{4}\left(3 e^{i \phi_{1}}+e^{i \phi_{0}}\right)$ and $b=\frac{1}{4}\left(e^{i \phi_{1}}-e^{i \phi_{0}}\right)$. It can be shown that a hydrogen atom undergoing a radiative transition from the $(F=1$, $\left.m_{F}=0\right)$ to the $\left(F=0, m_{F}=0\right)$ state has a probability $p_{1}=a^{*} a=\frac{5}{8}+\frac{3}{8} \cos \left(\phi_{1}-\phi_{0}\right)$ of being unperturbed by an exchange collision which occurs at a random time during the oscillation period of the cavity field. ${ }^{14}$ If the second atom is hydrogen, there is an additional probability $p_{2}=\operatorname{Re}\left(a^{*} b\right)=b^{*} b=\frac{1}{8}-\frac{1}{8} \cos \left(\phi_{1}-\phi_{0}\right)$ that the first atom leaves and the second atom enters the radiative state. It is this event which distinguishes hydrogen-hydrogen and hydrogen-nonhydrogen collisions. If an electron is not exchanged, $\left(\phi_{1}-\phi_{0}\right)=0,2 \pi, 4 \pi \cdots$, then $p_{1}=1$ and $p_{2}=0$. If an electron is exchanged, $\left(\phi_{1}-\phi_{0}\right)$ $=\pi, 3 \pi, 5 \pi, \cdots$, then $p_{1}=\frac{1}{4}$ and $p_{2}=\frac{1}{4}$. If the col-

${ }^{24}$ A. Dalgarno, Proc. Roy. Soc. (London) 262, 132 (1961).

${ }^{25}$ See also A. E. Glassgold, Phys. Rev. 132, 2144 (1963). lisions are strong so that all phase shifts are large and random, then $p_{1}=\frac{5}{8}$ and $p_{2}=\frac{1}{8}$. For oxygen collisions, $a=\frac{1}{3}\left(2 e^{i \phi_{3} / 2}+e^{i \phi_{1 / 2}}\right)$, and $b=\frac{1}{6}\left(e^{i \phi_{3} / 2}-e^{i \phi_{1 / 2}}\right)$, so that $p_{1}=a^{*} a=(5 / 9)+(4 / 9) \cos \left(\phi_{3 / 2}-\phi_{1 / 2}\right)$, which is $1,1 / 9$, or $5 / 9$ for no exchange, exchange, or strong collisions, respectively. In computing $p_{1}$ and $p_{2}$, we have assumed that the collision occurs at a time $t_{1}$ and averaged the outcome of the collision with respect to $t_{1}$ over the hyperfine oscillation period. The outcome of an exchange collision depends on the initial hydrogen state, but the initial state of the radiating atom oscillates in time at the hyperfine frequency from one which is predominantly $\left(m_{S}=+\frac{1}{2}, m_{I}=-\frac{1}{2}\right)$ to one which is predominantly $\left(m_{S}=-\frac{1}{2}, m_{I}=+\frac{1}{2}\right)$. The average is required because the collisions occur at random times during the oscillation period. The final result is not at all obvious.

\section{EXPERIMENTAL TECHNIQUE}

\section{A. Modification of the Maser}

The measurements were made with the second hydrogen maser described briefly by Ramsey. ${ }^{5}$ A detailed paper treating construction and operation of hydrogen masers will appear elsewhere, ${ }^{15}$ so we include here only those features of special relevance to the present experiment.

Considerable care was taken to keep the vacuum system as clean as possible by isolating the mercury diffusion pumps from the forepump with a Zeolite $13-x \operatorname{trap}^{26}$ and by eliminating all organic material other than Viton-A (some O-rings in the source chamber) and nylon (the monofilament thread used to open the trap door). Although the background pressure in the storage bulb chamber was no more than $5 \times 10^{-9} \mathrm{~mm} \mathrm{Hg}$ and no change in relaxation rate could be demonstrated on cooling the pumps to increase this pressure to $10^{-7}$ $\mathrm{mm} \mathrm{Hg}$, it must be stressed that the system was not ultraclean. Materials used in construction included stainless steel, aluminum, titanium, copper, gold, brass, Pyrex, fused quartz, and soft and hard solder.

The trap door was designed to open into the storage bulb vacuum chamber. It was necessary to add a titanium getter pump to this chamber to suppress the background pressure of polarized atomic hydrogen, Fig. 4. Polarized atomic hydrogen which flows back into the bulb through the open door decreases the measured $T_{2} / T_{1}$ ratio. Without the titanium pump, a sizable maser signal was observed on sealing the state-selected beam bulb entrance and opening the door, even though there was no line-of-sight path from the state-selecting magnet to the door. With the titanium pump, the $T_{2} / T_{1}$ flow-back error was reduced to $-1 \%$. Before a series of experiments were run, the pump was activated for a few minutes by heating the titanium wire. No change in relaxation rate was observed during this procedure.

The state-selected hydrogen source was regulated to

${ }^{26}$ M. A. Biondi, Rev. Sci. Instr. 30, 831 (1959). 


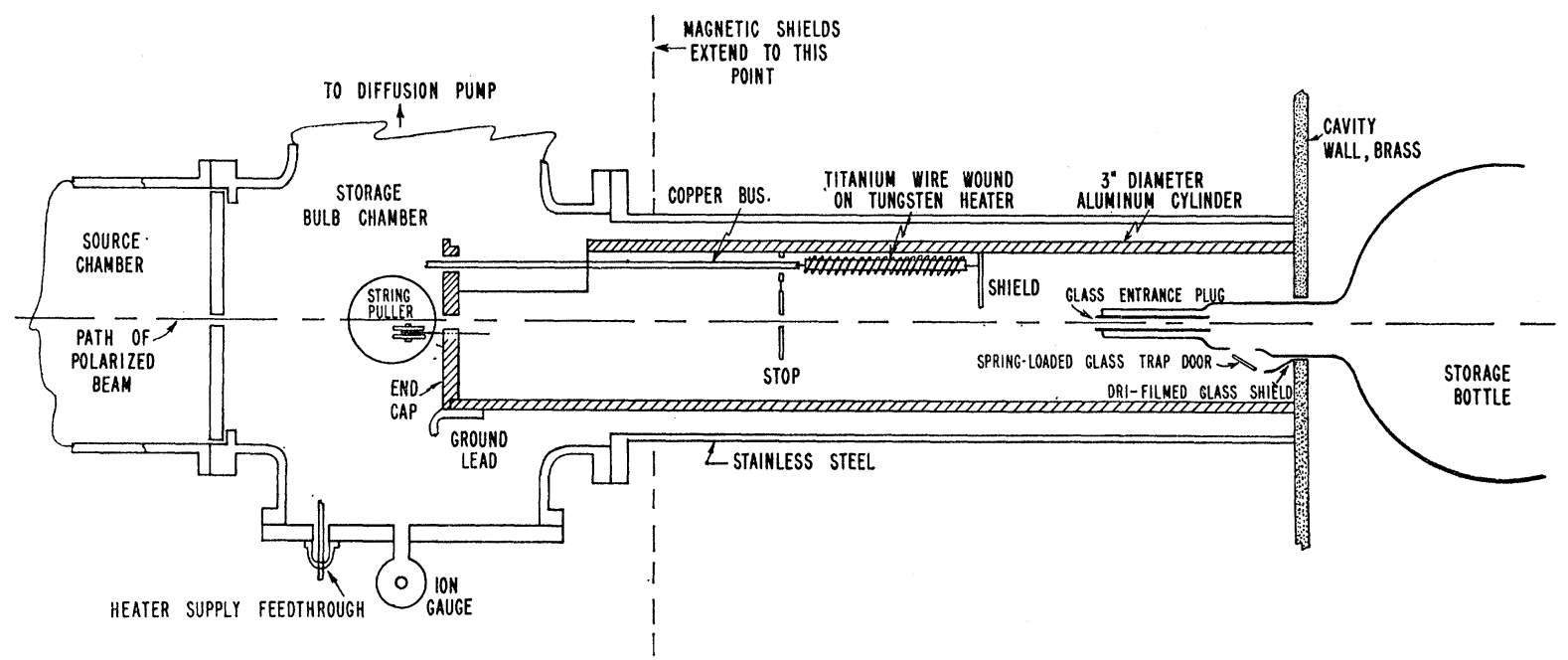

FIG. 4. Top view of the titanium pump assembly used in the trap-door experiments.

minimize any drift in beam intensity between measurements of the maser signals. This was done by inserting as an atom recombination detector one arm of a platinum-wire (0.0001 in.) resistance bridge into the beam near the source and by controlling the discharge power with a feedback system. Without the regulator, the beam intensity would drift about $10 \%$ per hour. With the regulator, the drift was less than $0.3 \%$ per hour.

Four different storage bulb surfaces were used. The first was a coating of dimethyldichlorosilane on fused quartz (GE Dri-Film SC-02). The second was a coating of a co-polymer of hexafluoropropylene and tetrafluoroethylene (du Pont FEP Teflon Product Code 120) which was exposed to Dri-Film. The third was an FEP coating contaminated with a substance emitted from a hot Viton-A O-ring. (Viton-A is a duPont co-polymer of vinylidene fluoride and hexafluoropropylene.) The fourth was FEP Teflon. The bulb entrance plugs and the trap door were coated either with Teflon or Dri-Film, and the tube leading from the added gas discharge to the bulb was coated with Teflon.

The cavity and magnetic shield assembly is shown schematically in Fig. 5 . The field used was about $5 \times 10^{-2}$

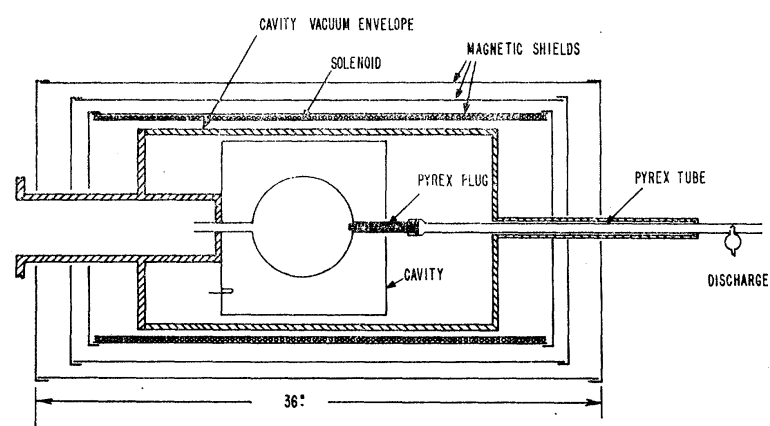

FIG. 5. Schematic view of the cavity and magnetic shields.
G. The operating points are indicated in Fig. 6, which shows total radiation decay rates and relative signal amplitudes as a function of the applied magnetic field. The relaxation rate behavior shown in the figure is consistent with the Zeeman relaxation rate theory developed for the maser: the rate goes to zero as the transition frequency between the states 1 and 2 or 2 and 3 exceeds the bounce frequency of the atoms in the bulb. ${ }^{4}$ The relative signal height can continue to change as a function of field at the higher fields if transitions occur before the atoms reach the bulb, but the outcome of the $T_{2} / T_{1}$ experiment does not depend on the composition of the beam. At low fields the relative signal

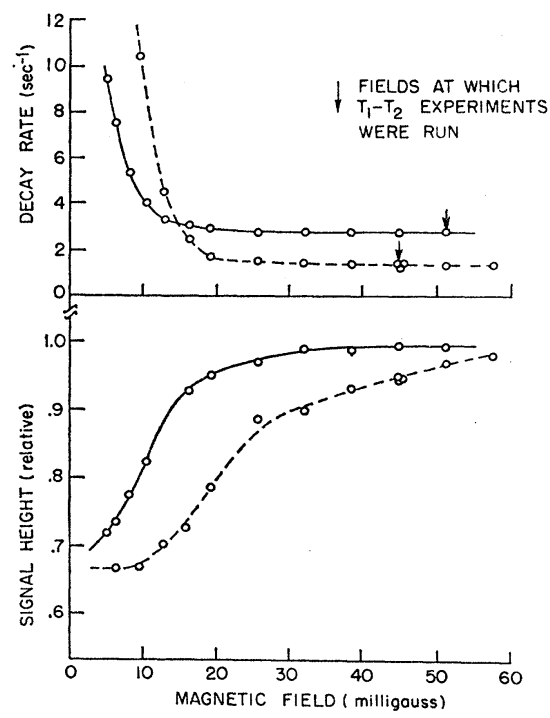

FIg. 6. Total decay rate and relative signal height as a function of magnetic field. The dashed curves are for an inner shield of Mumetal and the solid curves for one of Moly Permalloy. (Shield materials manufactured by the Allegheny Ludlum Steel Corporation.) 
heights approach $2 / 3$, which is the behavior expected for a state-selected beam for which $I_{1}=I_{2}$ and $I_{3}=I_{4}=0$.

Both Pyrex and fused quartz radiofrequency discharges were used to generate unpolarized atomic hydrogen and deuterium. The open end of the tube seen at the right of Fig. 5 was connected to a stainless-steel vacuum system pumped with a 15-liter/sec VacIon pump. (This system was modified in the course of the work and was ultimately made ultraclean, containing besides the pump only stainless steel, gold, fused quartz, and Pyrex.) The size of the discharge exit and the storage bulb connecting plug were chosen so that the pressure in the discharge was about $0.1 \mathrm{~mm} \mathrm{Hg}$ and the mean free path in the tube leading to the storage bulb was about the length of the tube at the fluxes required for the experiment. The gas was fed into the discharge through a palladium-silver leak. ${ }^{27}$ The maser relaxation rate did not change on increasing the $\mathrm{H}_{2}$ or $\mathrm{D}_{2}$ pressure if the discharge was off, and it did not decrease significantly on turning the discharge on if the leak was cold. In the latter case, only a surface discharge was observed. These measurements show that the storage bulbs were not contaminated by foreign material passing the palladium-silver leak or generated in the discharge. In changing from $\mathrm{H}_{2}$ to $\mathrm{D}_{2}$ or from $\mathrm{D}_{2}$ to $\mathrm{H}_{2}$, an exhaustive rinsing procedure was followed in which the leak was repeatedly baked under vacuum, cooled, and exposed to the new gas.

In a series of preliminary experiments a number of approximate absolute radiation decay-rate cross sections were measured by replacing the added gas discharge by a gas handling system and the connecting Pyrex plug by a fine capillary. The storage bulb pressure was measured as a function of a source chamber pressure, as read on a simple manometer, by connecting a McLeod gauge to the bulb. The calibration was made over a bulb pressure range for which the capillary flow was effusive $\left(1 \times 10^{-4}\right.$ to $\left.5 \times 10^{-4} \mathrm{~mm}\right)$, so that it could be extrapolated linearly to lower pressures. By this means, an absolute calibration was obtained which was independent of the size of the bulb or the McLeod gauge, so the latter could be removed for the pulse measurements.

In the $\mathrm{NO}$ and $\mathrm{O}_{2} T_{2} / T_{1}$ experiments, the gases were leaked directly into the main storage bulb vacuum chamber. The $\mathrm{H}-\mathrm{NO}$ and $\mathrm{H}-\mathrm{O}_{2}$ decay rate cross sections are so large that attenuation of the hydrogen beam by atom-molecule momentum transfer is negligible at the gas pressures required to quench the maser signal. Only those collisions which occur in the storage bulb are of importance because a hydrogen atom spends $10^{4}$ times as much time there as it does in the beam.

\section{B. Pulse Methods}

The electronic system delivers a short pulse to the cavity at the hydrogen hyperfine frequency of sufficient

${ }^{27}$ R. W. Compton and M. T. Elford, J. Sci. Instr. 39, 480 (1962); J. B. Hunter, Platinum Metals Rev. 4, 130 (1960). power to bring the atoms to the optimum radiating state. It then detects the energy emitted by the atoms and analyzes the signal for its decay time and its initial amplitude. These measurements can be made to within $1 \%$ when the maser is operated at a beam flux so small that the signals decay exponentially in time.

The general features of this system are shown in Fig. 7. A pulse at the hydrogen hyperfine frequency is generated by the modulator when the $29.6-\mathrm{Mc} / \mathrm{sec}$ gate is closed by a $0.003-\mathrm{sec}$ square wave. The pulse power is $10^{5}$ times larger than the maser signal power, so the pulse is delivered to the detector and to the cavity in such a way that the net signal arriving at the balanced mixer does not saturate the detector. The cavity is critically coupled to the line and tuned to the transition frequency by a standard reflection technique. The i.f. signal is amplified about $70 \mathrm{~dB}$ and converted to 5.76 $\mathrm{kc} / \mathrm{sec}$. The conversion is linear to well within $1 \%$. The bandwidth of the system is then limited to $150 \mathrm{cps}$ by a bandpass amplifier with a Lorentzian transfer function.

Both the amplitude and the initial shape of the signal are modified by the bandpass filter, but once the filter transient has had time to decay and provided the signal-to-noise ratio is sufficiently high, the output signal decays exponentially with the same time constant as the cavity oscillating magnetic field. If the decay constant analysis is delayed until the filter transient has decayed, then the initial amplitude can be obtained by extrapolating back in time. ${ }^{28}$ The analysis is more complicated at lower signal-to-noise ratios; the signalto-noise-ratio decreases as the signal decays.

An integration technique was developed in which the signals were repeatedly integrated over two successive periods of time. The first three segments of the upper traces in Fig. 2 monitor the analysis delay time and the first and second integration periods, respectively. By repeating the analysis for different integration

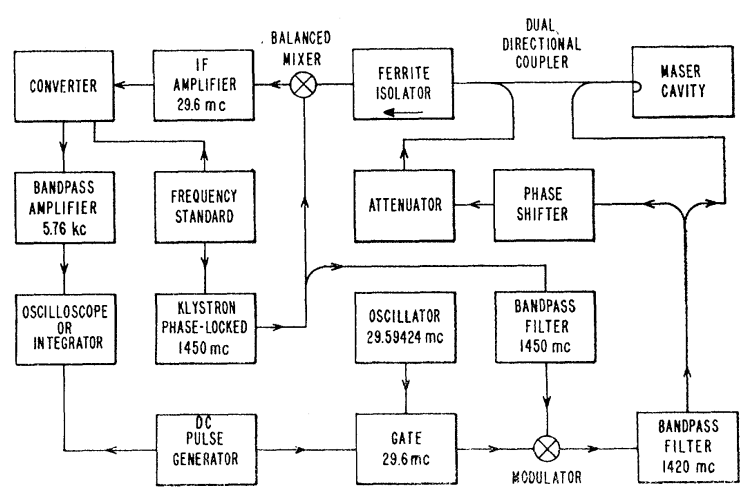

FIG. 7. Pulsed-signal detection system.

${ }^{28}$ The cavity oscillating-field amplitude following the pulse is strictly proportional to the steady-state hydrogen polarization density $n_{0}$ or $n$ only in the limit of vanishing pulse width. By extrapolating back to the beginning of the microwave pulse, the error in the measured $T_{2} / T_{1}$ ratios arising from the relaxation which occurs during the pulse is reduced to the order $0.1 \%$. 
periods, it is possible to detect any significant departure from simple exponential decay. (One can also take a multiple exposure photograph of an oscilloscope display of the output signal and fit the photograph with a standard curve, but this method is far less successful.) The details of the signal-to-noise theory and the integration technique are considered in another paper. ${ }^{16}$

\section{Measurement Procedure}

The choice of the maser operating point is of prime importance. If the hydrogen beam flux is too high, either the signals become nonexponential, or hydrogenhydrogen spin exchange occurs, or both occur, depending upon the value of the cavity and bulb parameters. If the signals are nonexponential, the amplitude-decay-rate analysis is not valid. If hydrogen-hydrogen spin exchange occurs, the wall-relaxation process is obscured and the trap door experiment is not a legitimate control. On the other hand, if the hydrogen-beam flux is too low, the signal is too small. In practice, the problem of nonexponential decay dominates, and the amplitude of the signal which can be obtained at the largest usable beam flux is proportional to $T_{0}{ }^{-1}$ and independent of all other cavity and bulb parameters (provided the cavity is critically coupled to the line). The experiment can be done well if $T_{0}^{-1}$ is greater than about $1.6 \mathrm{sec}^{-1}$; the bulb escape rate $T_{e}^{-1}$ is chosen accordingly.

The experiments were done in sets, one member of a set serving as a control on the spin-relaxation properties of the storabe bulb wall. A set consisted of as many experiments as could be performed consecutively in a few hours time. The pulse power was optimized and the behavior of the maser signal was studied as a function of beam flux at the beginning of each new set of measurements. The source was set and regulated at a flux at which the signal was exponential but below which any flux-dependent decay-rate change could be detected. This insured that the hydrogen-hydrogen spin-exchange relaxation rate was less than about $0.02 \mathrm{sec}^{-1}$. (A direct estimate was made of the background hydrogen density later by measuring the pulsed-signal amplitude at oscillation threshold.) In the main, only two operations were performed during a set: the added gas sources were turned up and down or on and off (or the trap door was opened and closed), and the i.f. amplifier gain was varied, as higher gain was required for the analysis of lower level signals. The relative gain was calibrated after each set of experiments by feeding a fixed low-noise signal into the amplifier through dummy crystals and by measuring the detector output with the integration system at each of the gain settings used.

A typical $T_{2} / T_{1}$ experiment could be completed in about $1 \mathrm{~h}$ and involved the analysis of about 15 maser signals. Measurements of $n$ and $T_{0}^{-1}+T_{g 2}{ }^{-1}$ were made at about 10 different added-gas pressures, and every 2 or 3 measurements of this kind were bracketed by measurements of $n_{0}$ and $T_{0}^{-1}$. (In Dri-Film bulbs the $n_{0}$ and $T_{0}^{-1}$ measurements were made only at the beginning of the experiment for reasons which will be apparent below.) The value of $n_{0}$ used in computing a given $n_{0} / n$ was interpolated from the two adjacent $n_{0}$ measurements, assuming any drift in $n_{0}$ to be linear in time. The value of $T_{0}^{-1}$ used in computing $T_{0} / T_{g 2}$ was the average value of all the $T_{0}{ }^{-1}$ measurements. The various $\left(n_{0} / n, T_{0} / T_{g 2}\right)$ points were plotted (see Figs. 3, 9, and 10) and a least-square fit was made to the line passing through the point $(1,0)$, assuming the values of $n_{0} / n$ to be normally distributed at fixed values of $T_{0} / T_{g 2}$. The slope and the $95 \%$ confidence interval for the slope were computed. The computations and curve plotting were done on an IBM 7094 computer.

\section{RESULTS AND INTERPRETATION}

The data are tabulated in detail in Ref. 16, and we will try to condense the essentials of that effort here. The surface phenomena are considerably more complex than formerly supposed. ${ }^{4,29}$ There appear to be two kinds of wall-relaxation processes, one which we have allowed for in Sec. II, which is a fixed process, and another which depends on the density of the atomic hydrogen. The fixed process is successfully dealt with by the trap door control, but the density-dependent process is not. Whereas the deuterium, nitric oxide, and oxygen $T_{2} / T_{1}$ results agree beautifully with the theory, the hydrogen values are low. But there is no doubt about the existence of the atom-to-atom coherence effect: the hydrogen-hydrogen $T_{2} / T_{1}$ ratio is at least 1.9 .

In the paragraphs that follow, we present the $T_{2} / T_{1}$ results and discuss the density-dependent and the density-independent wall relaxation. We conclude with the measurements of the absolute radiation decay rate cross sections of $\mathrm{NO}$ and $\mathrm{O}_{2}$.

\section{A. $T_{g 2} / T_{g 1}$ Ratios}

We plot in Fig. 8 the results based on the relative slopes of the experiments in a single set, i.e., the $T_{g 2} / T_{g 1}$ values which are independent of any fixed wall-relax-

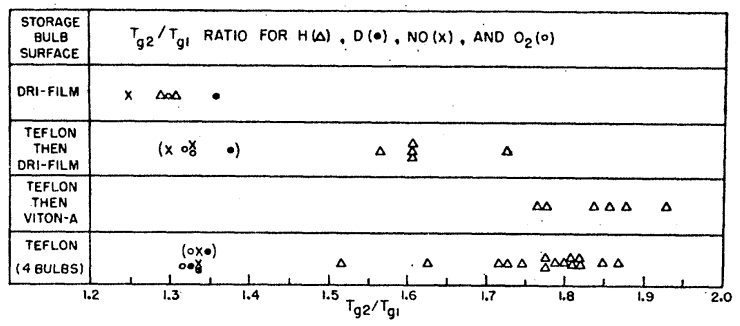

Fig. 8. $T_{2} / T_{1}$ ratios for $\mathrm{H}, \mathrm{D}, \mathrm{NO}$, and $\mathrm{O}_{2}$. The bulb surfaces have been described in Sec. III A. The results in parentheses were plotted by assigning the relative slope 1.33 to the experiment with the smallest confidence interval; a trap-door control was not available for these sets. NO was used as a control for many of the hydrogen experiments.

${ }^{29}$ H. C. Berg and D. Kleppner, Rev. Sci. Instr. 33, 248 (1962). 


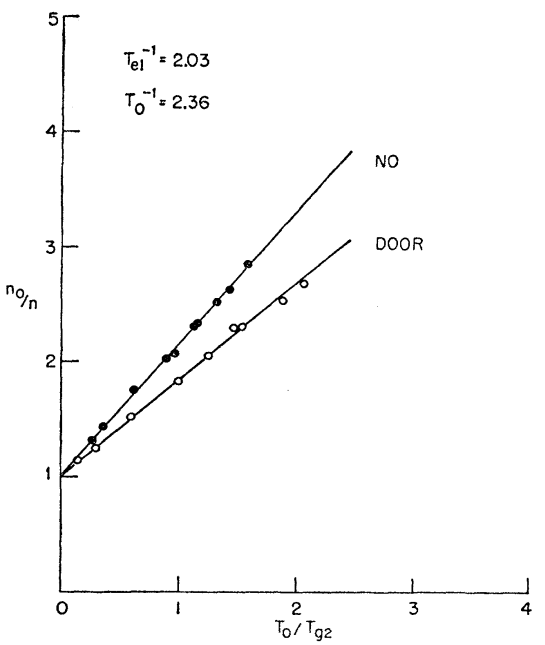

Fig. 9. The data for a set of experiments done with Teflon bulb 2. The slopes and $95 \%$ confidence intervals are $\mathrm{NO}=1.15 \pm 0.01$, door $=0.86 \pm 0.01$ (corrected). Relative to door $=1, \mathrm{NO}=1.34$.

ation process. ${ }^{30}$ The data from two sets are shown in Figs. 9 and 10.

The measurements made in Teflon bulbs are by far the most reliable for the following reasons. With Teflon, no changes in the wall can be attributed to the added gas once the gas has been removed. When the source is turned off, the decay rate returns promptly to its initial value. Any experiment can be repeated during a set, and the results always agree to within the confidence limits of the data. There is no dependence on the order of the addition of the gases. Small day-to-day changes in the absolute slope measured for the nonhydrogen gases are observed, even if the vacuum system has not been disturbed. Large day-to-day changes in the hydrogen slopes occur, and this is the main source of the scatter in the hydrogen results for a given surface. In all cases, the $95 \%$ confidence interval remains small, typically \pm 0.04 .

With Dri-Film, the added gases do cause a marked long-term change in the wall. Following the addition and removal of $\mathrm{H}$ and $\mathrm{D}$, the relaxation rate $T_{0}^{-1}$ remains larger than its initial value for several minutes; following the addition and removal of $\mathrm{NO}$ and $\mathrm{O}_{2}$, it remains smaller than its initial value for about $1 \mathrm{~h}$. For this reason, the Dri-Film bulbs were soon abandoned.

The $\mathrm{D}, \mathrm{NO}$, and $\mathrm{O}_{2}$ ratios each agree with the theoretical value $4 / 3$. While this result allows us to rule out the relaxation of hydrogen by direct chemical reaction with these gases, it does not tell us whether

${ }^{30}$ Three small corrections have been applied to the slopes, each based on a measured quantity. The trap-door slopes have been increased $1 \%$ to allow for polarized atom flow-back, Sec. III A, and about $1 \%$ to allow for the decrease in the number of hydrogenhydrogen collisions, Sec. III C. The hydrogen slopes have been decreased about $3 \%$. The added hydrogen has a small negative magnetization $\left(I_{2}<I_{4}\right)$ which can be measured by pulsing the maser with the added hydrogen beam on and the state-selected beam off. The correction is inversely proportional to the maser signal level. spin-exchange collisions occur with free radicals generated or adsorbed at the wall. (By "free radical," we mean any atom or molecule with an unpaired electron.)

The hydrogen $T_{2} / T_{1}$ ratios are smaller than the theoretical value 2 , and they vary widely from surface to surface. There is no doubt that this variation arises from a property of the bulb wall. Neither $2 S$ atoms nor $c^{3} \Pi_{u}(\nu=0)$ metastable molecules should be generated by the added-hydrogen source to a measurable extent. ${ }^{31,32}$ A great deal of work was done to rule out the possibility of any other source of contamination of the addedhydrogen beam.

\section{B. Hydrogen Density-Dependent Wall Relaxation}

The hydrogen results can be explained in a simple and direct way if the atoms collide with active sites on the wall generated by the adsorption or chemical reaction of other hydrogen atoms. If the number of these sites is proportional to the hydrogen density and the $T_{2} / T_{1}$ ratio for the active site relaxation process is less than 2 , then the $T_{2} / T_{1}$ plots are straight lines and the slopes are less than 2 . The rate of site formation depends on the detailed chemical state of the wall, and it is not surprising that the hydrogen results drift from day to day (the vacuum system is not ultraclean) and from bulb to bulb. Since deuterium and hydrogen are chemically similar we should expect a deuterium density-

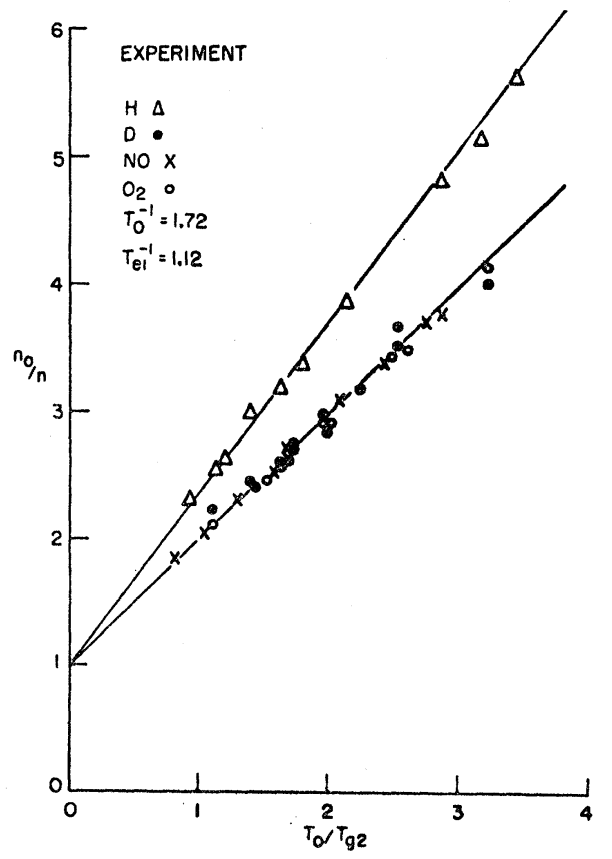

FIG. 10. The data for a set of experiments done with Teflon bulb 3. The slopes and $95 \%$ confidence intervals are $\mathrm{H}=1.34 \pm 0.07$ (corrected), $\mathrm{D}=1.01 \pm 0.06, \mathrm{NO}=1.00 \pm 0.04$, and $\mathrm{O}_{2}=0.99 \pm 0.01$. Relative to $\mathrm{O}_{2}=1.33, \mathrm{H}=1.80, \mathrm{D}=1.35, \mathrm{NO}=1.34$.

${ }^{31}$ W. Lichten, Phys. Rev. 120, 848 (1960); 126, 1020 (1962) ${ }^{32}$ W. E. Lamb, Jr., and R. C. Retherford, Phys. Rev. 79, 549 (1950). 
dependent effect. Since no divergence in the D, NO, and $\mathrm{O}_{2}$ results is seen, the active site $T_{2} / T_{1}$ value must be nearly $4 / 3$. (It could be as small as 1.20 , and the deuterium results in Teflon would only be $2 \%$ low.) The $T_{g 2} / T_{g 1}$ hydrogen ratio must then be at least as large as the largest value observed, Fig. 8.

Let the $T_{1}$ and $T_{2}$ times associated with the densitydependent surface effect be $T_{s 1}$ and $T_{s 2}$, and the times associated with the hydrogen gas phase relaxation be $T_{h 1}$ and $T_{h 2}$. The change observed in the decay rate on adding hydrogen is $T_{g 2}{ }^{-1}=T_{h 2}{ }^{-1}+T_{s 2}{ }^{-1}$. The inverse times are all proportional to $n_{\mathrm{H}} \cdot T_{s 1}{ }^{-1}$ and $T_{s 2}{ }^{-1}$ are proportional, as well, to the rate at which a given hydrogen atom strikes the wall, $\bar{v}_{\mathrm{H}} / \lambda$, where $\bar{v}_{\mathrm{H}}$ is the average hydrogen velocity and $\lambda$ is the mean free path in the bulb. $\left[\bar{v}_{\mathrm{H}}=(8 k T / \pi m)^{1 / 2}\right.$, where $k$ is Boltzmann's constant, $T$ is the absolute temperature, and $m$ is the mass of hydrogen atom.] If we include this process in the theory of Sec. II and divide out the fixed wall-relaxation effect by a control experiment in the usual way, the relative slope can be shown to be

$$
\left(\frac{T_{2}}{T_{1}}\right)_{\mathrm{rel}}=\left(\frac{T_{\mathrm{s} 2}}{T_{\mathrm{s} 1}}\right) r+\left(\frac{T_{h 2}}{T_{h 1}}\right)(1-r),
$$

where

$$
r=T_{s 2}{ }^{-1} /\left(T_{s 2}{ }^{-1}+T_{h 2^{-1}}\right)
$$

is independent of $n_{\mathrm{H}}$. If $T_{h 2} / T_{h 1}=2, T_{s 2} / T_{s 1}=4 / 3$, and $\left(T_{2} / T_{1}\right)_{\text {rel }}=1.80$ (the appropriate values for Teflon), then $r=0.30: 30 \%$ of the added-gas decay rate is induced at the wall. The relaxation in the Dri-Film bulb, on the other hand, is completely dominated by the wall.

Unfortunately, $\left(T_{2} / T_{1}\right)_{\mathrm{rel}}$ is very insensitive to $\lambda$, and the density-dependent effect cannot be demonstrated readily by using bulbs of different size. For a given $n_{\mathrm{H}}, T_{s 2}{ }^{-1} / T_{h 2^{-1}}=r /(1-r)$ is independent of $n_{\mathrm{H}}$. If we hold $n_{\mathrm{H}}$ constant and halve the diameter of the bulb, $T_{h 2^{-1}}$ remains constant and $T_{s 2}{ }^{-1}$ doubles; the structure of the wall remains the same, but the mean free path is halved. For $r=0.3, r /(1-r)=3 / 7$. For $r /(1-r)=6 / 7, r=6 / 13$, and $\left(T_{2} / T_{1}\right)_{\mathrm{rel}}=1.69$, which is only $6 \%$ smaller than the large bulb value. Teflon bulbs of various $\lambda$ were used, but $r$ was only changed by a factor 1.6, and any difference in relative slope was smaller than the scatter in the data.

Suppose a fraction of wall collisions $p$ lead to the formation of active sites which live a mean time $\tau$ and then spontaneously heal. How large is $\tau$ if a fraction $r$ of the relaxation rate $T_{g 2}{ }^{-1}$ arises from collisions at the wall? (In the next section we will see that if the sites generated by one hydrogen atom are removed only by the interaction of another hydrogen atom, the number of active sites is independent of the hydrogen density.) For simplicity, assume that the atoms all move with the same velocity $v_{\mathrm{H}}$. From Eqs. (11) and (18), and from the relation $v_{\mathrm{HH}}=\sqrt{2} v_{\mathrm{H}}$, we have

$$
T_{\mathrm{s} 2}-1 \simeq \frac{r n_{\mathrm{H} v_{\mathrm{H}} \sigma_{\mathrm{SF}}}}{\sqrt{2}(1-r)} .
$$

The number of active sites per $\mathrm{cm}^{2}$ of surface is $p \tau$ times the hydrogen flux $\frac{1}{4} n_{\mathrm{H}} v_{\mathrm{H}}$. If the active site is a free radical, the radiation decay rate cross section of the site is of order $3 \sigma_{\mathrm{SF}} / 4$. A given atom strikes the wall $v_{\mathrm{H}} / \lambda$ times per second, and it may hop from spot to spot $N$ times before it leaves the surface, therefore,

$$
T_{s 2}{ }^{-1} \simeq 3 n_{\mathrm{H}^{2} \mathrm{H}^{2} p \tau N \sigma_{\mathrm{SF}} / 16 \lambda .}
$$

Equating Eqs. (19) and (20), we find

$$
\tau \simeq 8 \sqrt{2} r \lambda / 3(1-r) p N v_{\mathrm{H}} .
$$

For $r=0.3, \lambda=10 \mathrm{~cm}, p=10^{-5}$ (there are at least $10^{5}$ adiabatic collisions at the Teflon wall), $N=1$, and $v_{\mathrm{H}}$ $=2.5 \times 10^{5} \mathrm{~cm} / \mathrm{sec}, \tau \simeq 5 \mathrm{sec}$. The fraction of the surface area which is active is quite small: at a hydrogen density for which $T_{h 2}=1 \mathrm{sec}, 3 n_{\mathrm{H} v_{\mathrm{H}}} p \sigma_{\mathrm{SF}} / 16 \simeq 10^{-4}$. In practice, if $\tau$ is not small compared to $T_{e 1}$, its effect should be observed on turning off the added hydrogen source: the signal decays back to its initial level with the two time constants $T_{e 1}$ and $\tau$. As noted in Sec. IV A, we observe this behavior with Dri-Film bulbs.

\section{Fixed Wall Relaxation}

At beam levels for which $T_{h 2^{-1}}$ is negligible, the density-dependent effect is negligible; no change in $T_{0}{ }^{-1}$ is seen on changing the intensity of the stateselected beam. Yet the values of $T_{0}^{-1}$ are larger than the values of $T_{e 1}^{-1}$ computed from the results of Clausing's calculations on the effusion of gases in cylindrical tubes.,21,22,33 The difference must arise from a fixed or density-independent wall-relaxation process, Table II. The mean number of wall collisions which occur in the relaxation time $T_{w 2}$ range from $7 \times 10^{3}$ for the Dri-Film bulb to $1.1 \times 10^{5}$ for Teflon bulb $4 . .^{34}$

The $T_{w 2} / T_{w 1}$ ratios given in Table II for Teflon are larger than can be explained by chemical or spinexchange relaxation. The values are sensitive to the measured slopes and to the difference between $T_{0}{ }^{-1}$ and $T_{e 1^{-1}}$, especially when the difference is small. The values in the last column hold if $T_{e 1}^{-1}$ is actually half as large as the value computed from the results of Clausing. (Unless the escape rates prove to be wrong, there is no justification for this choice.) The qualitative differences from surface to surface are clear. Compare the change in $T_{w 2} / T_{w 1}$ with the magnitude of the densitydependent effect, as judged by the hydrogen results of

${ }^{33} \mathrm{We}$ have not checked the plug escape rates experimentally, but it is easy to do with the maser by comparing tubes and thin holes.

${ }^{34}$ We have observed up to $3 \times 10^{3}$ collisions with fused quartz and $4 \times 10^{4}$ with a polychlorotrifluoroethylene wax (Halocarbon Products Corporation). 
TABLE II. Summary of data on fixed wall relaxation. The bulbs were spherical; the mean free path is $2 / 3$ the diameter. $T_{e 1}{ }^{-1}$ is computed from the exit plug radius $r$, Clausing's flow factor $K$, the volume of the bulb $V_{b}$, and the average velocity of atomic hydrogen $\bar{v}_{\mathrm{H}} ; T_{e 1}{ }^{-1}=\pi r^{2} K \bar{v}_{\mathrm{H}} / 4 V_{b} . T_{0}^{-1}$ is the average of the measured values. $T_{w 2} 2^{-1}=T_{0}^{-1}-T_{e 1}{ }^{-1}$. The equivalent door slope is the average of the measured door and $\mathrm{D}, \mathrm{NO}, \mathrm{O}_{2}$ slopes, but the latter have been divided by $4 / 3 . T_{w 2} / T_{w 1}$ is computed from Eq. $(9)$ assuming $\left(T_{2} / T_{1}\right)$ door $=1$. $\left(T_{w 2} / T_{w 1}\right)^{*}$ is computed in similar fashion, but the value used for $T_{e 1}^{-1}$ is a quantity 0.5 as large as the computed value of column four. The background hydrogen-hydrogen exchange relaxation rate was not well known for the Teflon-then-Dri-Film bulb, so the equivalent door slopes may be low; the three entries refer to days 1-2, 3-5, and 6-8 after the exposure of the bulb to Dri-Film. The Viton-A contamination levels of the three entries for that suiface are not necessarily the same.

\begin{tabular}{|c|c|c|c|c|c|c|c|c|}
\hline Storage-bulb surface & $\begin{array}{l}\text { Bulb mean } \\
\text { free path } \\
(\mathrm{cm})\end{array}$ & $\begin{array}{c}\text { Temp. } \\
\left({ }^{\circ} \mathrm{K}\right)\end{array}$ & $\begin{array}{c}T_{e 1^{-1}} \\
\left(\mathrm{sec}^{-1}\right)\end{array}$ & $\begin{array}{c}T_{0}^{-1} \\
\left(\mathrm{sec}^{-1}\right) \\
\end{array}$ & $\begin{array}{c}T_{w 2^{-1}} \\
\left(\mathrm{sec}^{-1}\right)\end{array}$ & $\begin{array}{c}\text { Equivalent } \\
\text { door slope }\end{array}$ & $\frac{T_{w 2}}{T_{w 1}}$ & $\left(\frac{T_{w 2}}{T_{w 1}}\right)^{*}$ \\
\hline Dri-Film & 10.3 & 296 & 1.12 & 4.58 & 3.46 & 0.92 & 1.1 & 1.1 \\
\hline Teflon then Dri-Film & 10.7 & 296 & 0.93 & $\begin{array}{l}1.37 \\
1.40 \\
1.43\end{array}$ & $\begin{array}{l}0.42 \\
0.47 \\
0.50\end{array}$ & $\begin{array}{l}0.95 \\
0.88 \\
0.74\end{array}$ & $\begin{array}{l}1.2 \\
1.4 \\
2.0\end{array}$ & $\begin{array}{l}1.1 \\
1.2 \\
1.4\end{array}$ \\
\hline Teflon then Viton-A & 6.5 & $\begin{array}{l}289 \\
308 \\
366\end{array}$ & $\begin{array}{l}1.73 \\
1.80 \\
1.97\end{array}$ & $\begin{array}{l}3.33 \\
3.60 \\
5.21\end{array}$ & $\begin{array}{l}1.60 \\
1.80 \\
3.24\end{array}$ & $\begin{array}{l}1.00 \\
1.00 \\
0.95\end{array}$ & $\begin{array}{l}1.0 \\
1.0 \\
1.1\end{array}$ & $\begin{array}{l}1.0 \\
1.0 \\
1.1\end{array}$ \\
\hline Teflon $\begin{array}{r}1 \\
2 \\
3 \\
4\end{array}$ & $\begin{array}{r}6.5 \\
10.3 \\
10.7 \\
10.7\end{array}$ & $\begin{array}{l}296 \\
296 \\
296 \\
294 \\
331 \\
363\end{array}$ & $\begin{array}{l}1.76 \\
2.03 \\
1.82 \\
1.83 \\
1.98 \\
2.04\end{array}$ & $\begin{array}{l}2.98 \\
2.34 \\
2.05 \\
2.04 \\
2.33 \\
2.52\end{array}$ & $\begin{array}{l}1.22 \\
0.31 \\
0.23 \\
0.21 \\
0.35 \\
0.48\end{array}$ & $\begin{array}{l}0.77 \\
0.86 \\
0.88 \\
0.86 \\
0.87 \\
0.82\end{array}$ & $\begin{array}{l}1.7 \\
2.2 \\
2.2 \\
2.6 \\
2.0 \\
2.2\end{array}$ & $\begin{array}{l}1.3 \\
1.2 \\
1.2 \\
1.3 \\
1.2 \\
1.3\end{array}$ \\
\hline
\end{tabular}

Fig. 8. Note that Viton-A contamination suppresses the density-dependent effect, increases the fixed wallrelaxation rate, and leads to the $T_{w 2} / T_{w 1}$ value 1 . As the Teflon-then-Dri-Film wall ages, the nature of the surface relaxation changes markedly, but the change in $T_{w 2}{ }^{-1}$ is small.

The following chemical process is characterized by a

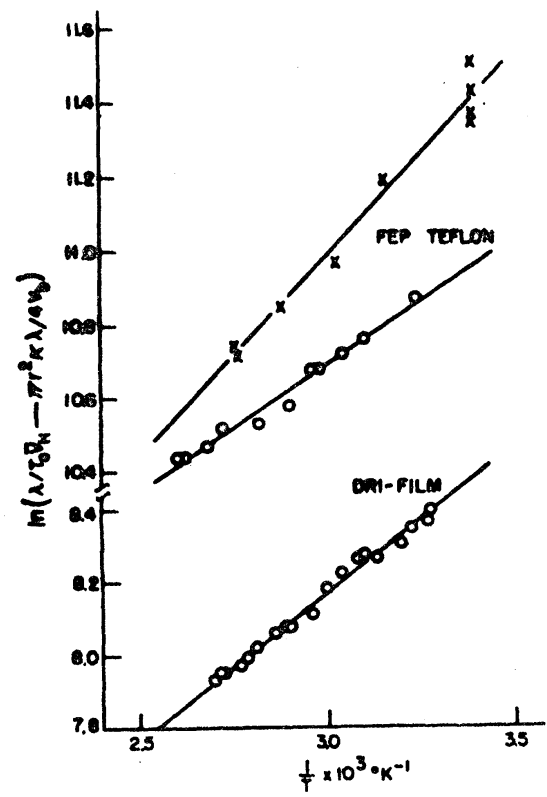

FIg. 11. Fixed wall relaxation rates as a function of temperature fit to the model $\ln \left(\lambda / \bar{v}_{\mathrm{H}} T_{w 2}\right)=-b / k T+\ln a$. Reading from top to bottom: $a=6 \times 10^{-4}, b=0.1 \mathrm{eV}$ (Teflon bulb 4); $a=2 \times 10^{-4}$, $b=0.07 \mathrm{eV}$ (a single exit bulb for which $T_{w 2}{ }^{-1}=0.46 \mathrm{sec}^{-1}$ at $296^{\circ} \mathrm{K}$ ) $; a=2.6 \times 10^{-3}, b=0.07 \mathrm{eV}$ (a single neck bulb for which $T_{w 2}{ }^{-1}=5.48 \mathrm{sec}^{-1}$ at $305^{\circ} \mathrm{K}$ ). fixed wall relaxation rate and a $T_{w 2} / T_{w 1}$ value between 1 and 4/3. The first event is the formation of a free radical by the reaction of hydrogen with a molecule of the wall $R H$ :

$$
\mathrm{H} \cdot+R \mathrm{H} \rightarrow R \cdot+\mathrm{H}_{2} .
$$

(We use a dot to indicate an unpaired electron.) The hydrogen molecule is of no further interest. Another hydrogen atom may spin exchange or react with $R$. restoring the wall:

$$
\mathrm{H} \cdot+R \cdot \rightarrow R \mathrm{H}
$$

Let the fraction of the wall area which is $R H$ be $f_{1}$ and the fraction which is $R \cdot$ be $f_{2}$. Let the probability of the first reaction be $p_{1}$ for a collision between $H \cdot$ and $R \mathrm{H}$, and the probability for the second reaction be $p_{2}$ and for spin exchange be $p_{3}$ for a collision between $\mathrm{H}$ - and $R \cdot$. The net rate of $R \cdot$ formation is $\frac{1}{4} n_{\mathrm{H}} v_{\mathrm{H}} A\left(p_{1} f_{1}-p_{2} f_{2}\right)$, where $A$ is the area of the wall. The steady-state value of $f_{2}$ is $p_{1} f_{1} / p_{2}$. Since the vast majority of collisions are adiabatic, $f_{1} \simeq 1$, and $f_{2} \simeq p_{1} / p_{2}$. The total relaxation rate is

$$
\begin{aligned}
T_{w 2}{ }^{-1} & =\left(v_{\mathrm{H}} / \lambda\right)\left(p_{1} f_{1}+p_{2} f_{2}+\frac{3}{4} p_{3} f_{2}\right) \\
\simeq & \simeq\left(2 v_{\mathrm{H}} p_{1} / \lambda\right)\left[1+\frac{3}{8}\left(p_{3} / p_{2}\right)\right] .
\end{aligned}
$$

In the limit that $p_{3} \gg p_{2}, T_{w 2} / T_{w 1}=4 / 3$. In the limit $p_{3} \ll p_{2}, T_{w 2} / T_{w 1}=1$.

As has been pointed out before, ${ }^{4,31}$ the value of $p_{1}$ is sufficiently large for the reaction between atomic hydrogen and paraffin hydrocarbons to make this mechanism a reasonable one for relaxation on Dri-Film. The commercial Teflon used in these experiments contains wetting agents, and there is no reason to suppose it is pure fluorocarbon. If it were, $p_{1}$ should be far too small. (We have also observed that at least half again 
as much $\mathrm{H}$ - must be added to a Dri-Film bulb than to a Teflon bulb to attain the same value of $T_{h 2}{ }^{-1}$, other conditions being the same, which is direct evidence of the conversion of $\mathrm{H}$ - to $\mathrm{H}_{2}$. If tritium were used, the wall should become progressively more radioactive.)

The temperature dependence of $T_{w 2}{ }^{-1}$ was studied over the range $273-373^{\circ} \mathrm{K}$ by heating the cavity and bulb assembly and measuring $T_{0}^{-1}$. The data can be fitted to the model

$$
T_{w 2}{ }^{-1}=\left(\bar{v}_{\mathrm{H}} / \lambda\right) a \exp (-b / k T),
$$

where $a$ and $b$ are numbers independent of $T$, Fig. 11 . From the results given in Fig. 11, we see that $a$ is of order $10^{-3}$ and $b$ is of order $0.1 \mathrm{eV}$. The wall relaxation rate increases with temperature more rapidly than the collision rate. Provided there is no gross structural change in the wall, this behavior cannot be explained if the atoms interact only with the local effective magnetic field of the wall, for the average time that the atom is adsorbed at the wall decreases with temperature. In Ref. 4, Eq. (58), $b$ is interpreted as a chemicalreaction activation energy, $E_{a}$, and it is assumed that an atom colliding with the wall will react with probability $P=(2 / 3)^{1 / 2} a$ if its initial kinetic energy is greater than or equal to $E_{a}$. When this interpretation was made, it was thought that most wall collisions were elastic, i.e., that adsorption times were small. Preliminary measurements on the temperature dependence of the wall-frequency shifts (FEP Teflon, assuming again that there is no gross structural change in the wall) suggest that the adsorption energy is of order $0.17 \mathrm{eV} .^{35}$ This means that an atom is trapped at the wall for a period of time long compared to the vibrational period of the atoms of the wall. It also means that the trapped atom vibrates in a direction normal to the wall several hundred times on the average before it breaks free. Clearly, the reaction may occur at any time while the atom is at the wall, provided the translational energy of the wall molecule-trapped atom collision is sufficiently high. Since the average time the atom spends on the wall is proportional to $\exp (E / k T)$, where $E$ is the adsorption energy, we expect $b$ to be a number smaller than the activation energy, $E_{a}$.

If the adsorption energy is of order $0.1 \mathrm{eV}$, then adsorption times approach $10^{-11} \mathrm{sec}$, and the relaxation mechanism proposed by Bouchiat becomes important. ${ }^{12}$ Bouchiat discusses the dipole-dipole interaction between the magnetic moment of the electron of the adsorbed atom (in her case, rubidium) and the magnetic moments of the nuclei of the wall (protons or deuterons). The relaxation rate $T_{w 1}{ }^{-1}$ is of order $\mu_{0}^{2} H^{2} \tau_{c} \tau_{s} / h^{2} \tau_{v}$, where $\mu_{0}$ is the Bohr magneton; $H$ is the average amplitude of the magnetic field at the electron due to the nuclei of the wall while the atom is adsorbed at a specific site on the wall; $\tau_{c}$ is the average time an atom spends at such a site; $\tau_{s}$ is the total adsorption time $N \tau_{c}$, where

${ }^{35}$ S. B. Crampton (private communication).
I N A TOMIC H M ASER

TABLE III. Approximate radiation decay-rate cross sections for several gases. (These are not spin-flip cross sections.)

\begin{tabular}{lr}
\hline Gas & Cross section $\left(\mathrm{cm}^{2}\right)$ \\
\hline $\mathrm{NO}$ & $(1.9 \pm 0.2) \times 10^{-15}$ \\
$\mathrm{O}_{2}$ & $(1.9 \pm 0.2) \times 10^{-15}$ \\
$\mathrm{NO}_{2}$ & $\sim 2 \times 10^{-15}$ \\
$\mathrm{NH}_{3}$ & $\sim 2.5 \times 10^{-17}$ \\
$\mathrm{CH}_{2} \mathrm{CH}_{2}$ & $<10^{-17}$ \\
$\mathrm{~N}_{2} \mathrm{O}$ & $<10^{-18}$ \\
$\mathrm{CO}$ & $<10^{-18}$ \\
$\mathrm{CO}$ & $<10^{-18}$ \\
$\mathrm{Xe}$ & $<10^{-20}$ \\
\hline
\end{tabular}

$N$ is the average number of hops which an atom makes from site to site before leaving the wall; and $\tau_{v}$ is the average bulb transit time. For $H=5 \mathrm{G}$ (the field $10^{-8}$ $\mathrm{cm}$ from a proton), $\tau_{c}=\tau_{s}=10^{-11} \mathrm{sec}$, and $\tau_{v}=4 \times 10^{-5}$ sec, $T_{w 1^{-1}} \simeq 10^{-2} \mathrm{sec}^{-1}$. It will be of interest to extend the theory of Bouchiat to the hydrogen maser and to compute the theoretical ratio $T_{w 2} / T_{w 1}$.

\section{Absolute Radiation Decay-Rate Cross Sections}

The approximate radiation decay-rate cross sections for a series of gases are given in Table III. The data for the $\mathrm{NO}$ and $\mathrm{O}_{2}$ measurements is shown in Fig. 12. A polytetrafluoroethylene coated bulb was used at room temperature (du Pont TFE Teflon Clear Finish 852-201). The signals were analyzed photographically. Upper limits are given for some of the cross sections, since the gas samples were not analyzed for oxygen. Nitrogen dioxide is the only gas studied which accumulates at the bulb wall: the cross section was estimated by extrapolating back to the time that the gas was first added. The decay rate cross section for gas $G$ was computed from the relation $\left(n_{G} \bar{v}_{G \mathrm{H}} T_{g 2}\right)^{-1}$, which ignores any dependence on the relative translational energy of the collision. The velocity-averaged $300^{\circ} \mathrm{K}$ hydrogenhydrogen spin-flip cross section computed by Dalgarno ${ }^{24}$ is $2.1 \times 10^{-15} \mathrm{~cm}^{2}$. From Eq. (6) and Table III we estimate the hydrogen-nitric-oxide spin-flip cross sec-

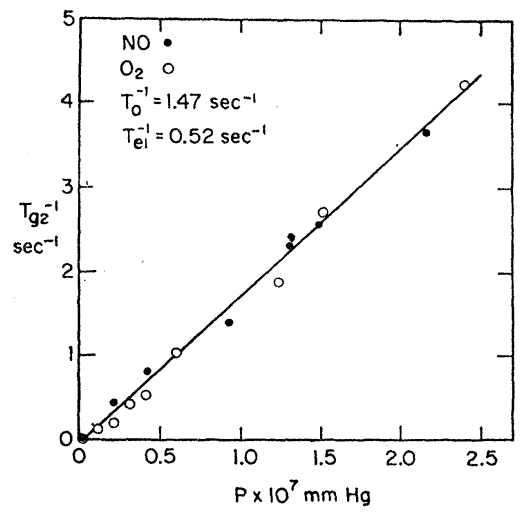

FIG. 12. Radiation decay rate increment $T_{g 2}{ }^{-1}$ as a function of the storage bulb pressure of added $\mathrm{NO}$ and $\mathrm{O}_{2}$. 
tion to be $2.5 \times 10^{-15} \mathrm{~cm}^{2}$. From Eq. (15) and Table III we estimate the hydrogen-oxygen spin-flip cross section to be $2.1 \times 10^{-15} \mathrm{~cm}^{2}$. These measurements are rather crude and could certainly be improved.

\section{CONCLUSION}

We have shown for the $\Delta m_{F}=0$ hyperfine transition of $1 S$ atomic hydrogen that a simple relationship exists between the rate that spin exchange collisions decrease the oscillating dipole moment of the radiating gas and the rate that they decrease the stationary-state population difference $\left(F=1, m_{F}=0\right)-\left(F=0, m_{F}=0\right)$. We have described a method of verifying this relationship with a pulsed-mode hydrogen maser experiment, which at the same time provides information on the spin relaxation of atoms at a solid surface.

For collisions with deuterium, nitric oxide, and oxygen, we have found the ratio for the $T_{2}$ and $T_{1}$ relaxation times to be very nearly $4 / 3$, in agreement with a theory which neglects spin-orbit coupling and all direct magnetic interactions. This is an important result, for the masses, nuclear moments, electronic angular momenta, and vibrational and rotational structures of these systems all differ. The relaxation process in the case of $\mathrm{H}-\mathrm{D}, \mathrm{H}-\mathrm{NO}$, and $\mathrm{H}-\mathrm{O}_{2}$ collisions must be strongly dominated by electron exchange. For collisions between free electrons and alkali atoms of large atomic number, it is not at all clear that this is true. ${ }^{11}$ To clarify the interpretation of the free-electron experiments, it will be of interest to do a careful (frequency shift)/(line broadening) ratio experiment with atomic hydrogen. We know in general that there must be coupling between the spin and orbital states, for otherwise, a system in spin exchange equilibrium is not in thermal equilibrium. ${ }^{7,36}$

For $\mathrm{H}-\mathrm{H}$ collisions we have found the ratio of the $T_{2}$ and $T_{1}$ relaxation times to be at least 1.9. Since this value is appreciably greater than $4 / 3$, we have confirmed that there is a spin correlation associated with the exchange interaction which transfers phase information from atom to atom.

Hydrogen atoms are relaxed at the wall of the storage bulb by at least two nonadiabatic processes. One, with a relaxation rate proportional to the hydrogen density,

36 This can be seen at once in the following way. The spinexchange transformation, Eq. (16), conserves the projection of the total electron-spin angular momentum. If we had a box of hydrogen atoms in the $\left(F=1, m_{F}=1\right)$ state, the atoms would remain in that state regardless of the number of exchange collisions, even though it is the state of highest energy. causes the measured hydrogen-hydrogen $T_{2} / T_{1}$ ratios to be smaller than the theoretical value 2 . The other, which is independent of the hydrogen density, has a relaxation rate which increases rapidly with temperature. The density-dependent process is particularly nasty, for it confuses any experiment on absolute spinexchange cross sections or (frequency shift)/(line broadening) ratios which might be performed with the maser.

Hydrogen masers are being built which are more nearly ultraclean, and there is hope that a systematic study of the relaxation rate and frequency shift properties of a number of surfaces can be made over a wide range of temperatures. We need to find a surface which is more chemically inert to atomic hydrogen, and we expect that a pure fluorocarbon will be. ${ }^{37}$ It will then be possible to study physical adsorption and the coupling between atomic hydrogen and the local effective magnetic field of the wall. If the maser is to be used only as a frequency standard, i.e., as a continuous wave oscillator on the $\Delta m_{F}=0$ transition, the present surfaces may be satisfactory: neither hydrogen-nonhydrogen spin-exchange collisions ${ }^{9,38}$ nor collisions which remove atoms by chemical reaction lead to a $\Delta m_{F}=0$ frequency shift. [Note added in proof. We have considered the magnetic relaxation process of Bouchiat. It may contribute to the fixed wall relaxation, but it cannot change the measured $T_{2} / T_{1}$ ratios or give rise to a hydrogen-density-dependent effect.]

\section{ACKNOWLEDGMENTS}

The author is indebted to Professor Norman F. Ramsey who supported and guided this research throughout its course. Design and construction of the second maser was a cooperative venture, involving the talents of Professor Daniel Kleppner, Hugh G. Robinson, Stuart B. Crampton, and Douglas Brenner. Contributions by Norval Fortson, Ekkehardt Recknagel, B. S. Mathur, and Than Myint are also acknowledged. Thanks are due to William Bossert, who wrote the computer program and introduced the author to the world of FORTRAN, and to David Pritchard, who assisted by reading many photographs.

${ }^{37}$ E. W. R. Steacie, Atomic and Free Radical Reactions (Reinhold Publishing Corporation, New York, 1954), 2nd ed., Vol. 2, Chap. 10.

${ }^{38}$ This has been shown experimentally with deuterium, S. B. Crampton, thesis, Harvard University, 1964 (unpublished). The hydrogen-hydrogen shift can be compensated for by a suitable cavity tuning procedure: see Ref. 15 and S. B. Crampton, D. Kleppner, and N. F. Ramsey, Phys. Rev. Letters 11, 338 (1963). 


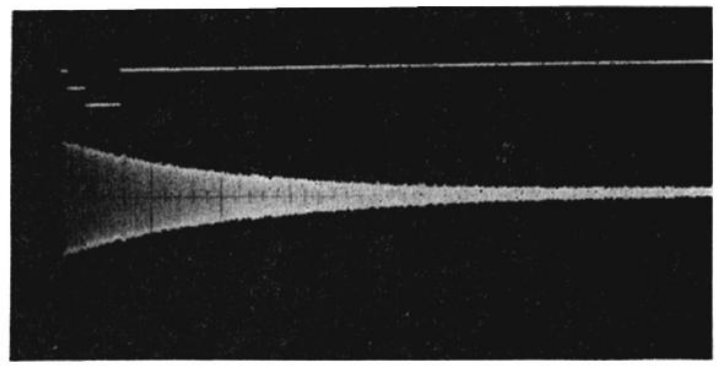

(a)

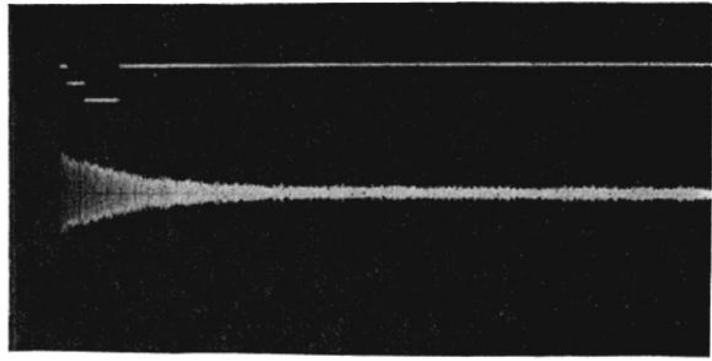

(b)

Fig. 2. (a) Decay of the maser signal without added gas. (The segments of the upper trace are $0.02,0.05$, and $0.10 \mathrm{sec}$ long, respectively. The carrier is $5.76 \mathrm{kc} / \mathrm{sec}$.) (b) As (a) except for the addition of unpolarized hydrogen and an increase in i.f. amplifier gain by a factor 1.8 . 